\title{
A primitive memory system for the deployment of transient attention
}

\author{
ÁRNI KRISTJÁNSSON and KEN NAKAYAMA \\ Harvard University, Cambridge, Massachusetts
}

\begin{abstract}
When transient attention is summoned by the sudden appearance of a large cue, it can be deployed to a small portion of the cue where a target appeared on previous trials (Kristjánsson, Mackeben, \& Nakayama, 2001). This result runs counter to the view that transient, or exogenous, attention is summoned automatically and indiscriminately to abruptly appearing stimuli. To further characterize the short-term learning mediating this phenomenon, we report the following results. (1) When there was a consistent relationship between a small identifying portion of the cue and the target, learning occurred rapidly. Thus, transient attention can be summoned to a distinctively colored or distinctively shaped portion of the cue as a consequence of repeated pairing (Experiments 1 and 2). (2) When there was a consistent relation between a given position on the object and its overall color or shape, no learning occurred (Experiments 3 and 4). Thus, transient attention cannot learn a complex relation between target position and shape or color. (3) We confirmed the fast object-centered learning of position shown in Kristjanssson et al. (2001). (4) Explicit knowledge of the cue-target relationship had no effect on the performance of the task. The results provide evidence for the existence of a primitive object-centered learning mechanism beneficial for the rapid deployment of transient attention. The possible role of such a mechanism in the maintenance of representations of the visual environment is discussed.
\end{abstract}

If an object suddenly appears in the visual field, visual attention is quickly and efficiently drawn to portions of the object that have proved important for behavior in the recent past (Kristjánsson, Mackeben, \& Nakayama, 2001). We showed in that paper that transient attention (a component of attention that is quickly applied to suddenly appearing stimuli; Nakayama \& Mackeben, 1989; see also Cheal \& Lyon, 1991) quickly "learns" a relationshipbetween a cue and a target in a visual discrimination task. This result was surprising, since many studies had indicated that when a behaviorally relevant stimulus suddenly appears, attention is allocated to it reflexively, without any form of higher order goal-driven properties (Jonides, 1981; Theeuwes, 1991; Yantis \& Jonides, 1984; see also discussions by Breitmeyer \& Ganz, 1976, and Lennie, 1980). According to this view, attention is allocated indiscriminately in a bottom-up fashion to sudden luminance onsets that are relevant to immediate behavior. Also, since the time course of this attentional deployment was very rapid and transient, reaching a peak at about $150 \mathrm{msec}$, we identified the learning as characteristic of transient attention (Kristjánsson et al., 2001; Nakayama \& Mackeben, 1989).

In the studies by Kristjánsson et al. (2001), performance on a discrimination task greatly improved if a target had a consistent spatial relationship to a cue. Thus, if the target

Á.K. was supported by a Fulbright graduate award, as well as an Eliot Grant from Harvard University. K.N. was supported by an AFOSR grant. Correspondence concerning this article should be addressed to Á. Kristjánsson, Institute of Cognitive Neuroscience, 17 Queen Square, London WCIN 3AR, England (e-mail: a.kristjansson@ucl.ac.uk). appeared a few times in a row on the left side of a much larger cue, performance improved dramatically, in comparison with cases in which the target appeared randomly at either end of the cue from one trial to the next. What turned out to be decisive for this learning to develop was whether there was a consistent location mapping between the cue and the target. Thus, if the target always appeared on the left part of the cue, performance was better than when it appeared at a random location on the cue. This result is inconsistent with a purely bottom-up model of attention shifts, since such an account would predict that attention would be grabbed automatically by the suddenly appearing cue and would be indiscriminately applied over its full extent. In the experiments of Kristjánsson et al. (2001), nothing in the perceptual appearance of the cue marked a region where the target was likely to appear. Importantly, the cue lead and target times we used were very brief, making it unlikely that any top-down, or explicit, strategies could aid performance (see also Nakayama \& Mackeben, 1989). Furthermore, the learning was object based, rather than space based, since it took place independent of the absolute location of the cue and the target.

Many studies have indicated that attentional capture can, in fact, be modulated by the expectancies of the observer (Folk \& Remington, 1999; Folk, Remington, \& Johnston, 1992; Luck \& Thomas, 1999; Theeuwes \& Burger, 1998; see also Yantis \& Jonides, 1996). Thus, the degree to which a suddenly appearing stimulus captured attention was partly dependent on how relevant it was to the task at hand or on prior experience. The study by Kristjánsson et al. (2001) demonstrated that prior experience can be decisively specific, determining exactly where, on a large ob- 
ject, exogenous attention will be deployed. The results of Kristjánsson et al. (2001) can, then, be considered further evidence for the adaptability of rapid shifts of attention, demonstrating that they need not be considered to be completely reflexive and bottom-up.

In this research, using our previous results as a starting point (Kristjánsson et al., 2001), we investigated what other relations between target location and a larger cue can be learned in the deployment of transient attention. We applied the methodology of our previous work to cases in which the relationship between the cue and the target depended on color (Experiment 1), on shape (Experiment 2), and also on a higher order relationship between the cue and the target, where the overall color (Experiment 3 ) or the overall shape (Experiment 4) of the cue indicated where on the cue the target would appear. The task in Experiments 3 and 4 was particularly challenging, since a two-step inference was required to learn the relationship between the cue and the target. First, the color (or shape) of the cue had to be identified, and then an inference about where on the cue the target would appear was needed. The tasks in Experiments 1 and 2 might have been easier to learn, since specific features on the cue indicated the target location.

We also sought to delineate the boundaries of this learning. Are there obvious contingencies that cannot be easily learned by the exogenous attentional system? Can transient attention be deployed to a specific position within an object (left or right) on the basis of differences in object shape or color? Our results in this article show that a simple one-step association between a feature of the cue (color or shape) can easily be learned by transient attention but also that a two-step contingency between the cue and the target cannot be learned. In addition, Experiments 5 and 6 showed that prior knowledge of the cue-target contingencies cannot be used by observers to improve performance, showing that the learning cannot be modulated by topdown strategies.

\section{EXPERIMENT 1 \\ Color Differences on a Cue Are Effective for Efficient Deployments of Transient Attention}

Do color differences on a cue lead to effective attentional deployments to the briefly presented cue? In other words, will performance on a discrimination task improve if the target always appears, for example, on the green half of a cue that is red on one side and green on the other? In our previous paper, a consistent location mapping between the cue and the target led to effective attentional deployments to a part of the cue (and subsequent target). Here, we investigate whether a similarly consistent relationship between the cue and the target, based on color, can facilitate discrimination performance. Kingstone and Klein (1991) investigated the effects of concurrent shape and position expectancy in a cuing paradigm that was, in some ways, reminiscent of the present paradigm, but in their experiment the cue stayed on for $1,500 \mathrm{msec}$, followed by the target, which means that their experiment did not address deployments of transient attention, as was the aim in the experiments described here.

The general design of the experiments in this paper is presented in Figure 1. The task of the observers was to indicate whether the eye target was looking up, down, to the left, or to the right, among distractors looking straight ahead (chance performance was thus $25 \%$ ). The bars seen in the figure cue the target location on each trial.

\section{Method}

There were five different conditions in the experiment. In the switch condition, the target alternately appeared at the red and the green ends of the cue from one trial to the next. In the random condition, the target appeared randomly at either end of the cue, unrelated to its color. In the streak condition, the target appeared at the same-colored part of the cue for long stretches of contiguous trials. ${ }^{1}$ In the consistent (red) and consistent (green) conditions, the target always appeared at the red or the green end of the cue within a block of trials. Which part of the cue was the red or the green part was determined randomly from one trial to the next under all five conditions, which means that there was no consistent location mapping between the cue and the target like that in our previous experiments (Kristjánsson et al., 2001). If transient attention can be directed to a particular color on the cue, performance should be best under the consistent and streak conditions, since it is only under these conditions that the color of a part of the cue consistently predicts the target location. The cue was $100 \%$ valid as to where the target would appear on the imaginary circle of target and distractors (see Figure 1).

Stimuli. The targets were eye-like stimuli (see Figure 1) that were looking to the left, to the right, up, or down, and the task of the observer was to indicate which way the eye was looking (chance performance was thus $25 \%$ ). The eyes consisted of a light gray $\left(41.0 \mathrm{~cd} / \mathrm{m}^{2}\right)$ ring surrounding a smaller disk of the same color (the eyeball ). On the targets, the eyeballs were looking to the left, to the right, up, or down, whereas on the distractors the eyeballs looked straight ahead. The diameter of the targets and the distractors was $1.5^{\circ}$ (at a viewing distance of $57 \mathrm{~cm}$ ). The cue was a pair of horizontal bars of a length equal to four times the diameter of the target. One half of the cue was green $\left(11.8 \mathrm{~cd} / \mathrm{m}^{2}\right)$, whereas the other half was red $\left(9.7 \mathrm{~cd} / \mathrm{m}^{2}\right.$; see Figure $\left.2 \mathrm{~A}\right)$. No attempt was made to disentangle the effects of luminance and color in this experiment, but note that the luminance differences between the two colors were very small. The display items appeared on a black $\left(0.50 \mathrm{~cd} / \mathrm{m}^{2}\right)$ background. The imaginary circle of target and distractors had a radius of $5^{\circ}$. Stimuli were presented on a CRT screen (with a frame rate of $75 \mathrm{~Hz}$ ), driven by an Apple Macintosh G3 computer. We used a local mask that replaced the target and the distractors. The mask was a set of square patches of random dots $\left(\right.$ size $\left.=6^{\prime}\right)$. Each side of the square patch was equal in length to the diameter of the target and the distractors. The VisionShell programming library was used to generate the stimuli (for information, go to http://www.visionshell.com).

Procedure. The observers were seated $57 \mathrm{~cm}$ from the screen. They were instructed to fixate on a cross at the center of the screen for the whole duration of each trial. A cue indicated the position of an upcoming target in an array of eight items. After the cue lead time, the target appeared, while the remaining positions on the imaginary circle were occupied by distractors. Two different combinations of cue lead and target times were tested for each observer (see Figure 2B). We used cue lead and target times at which the transient component of attention was maximally effective, which were, furthermore, too brief for any benefits from the activation of sustained attention (see Kristjánsson et al., 2001; Nakayama \& Mackeben, 1989). Furthermore, the cue lead time was also too brief to allow 


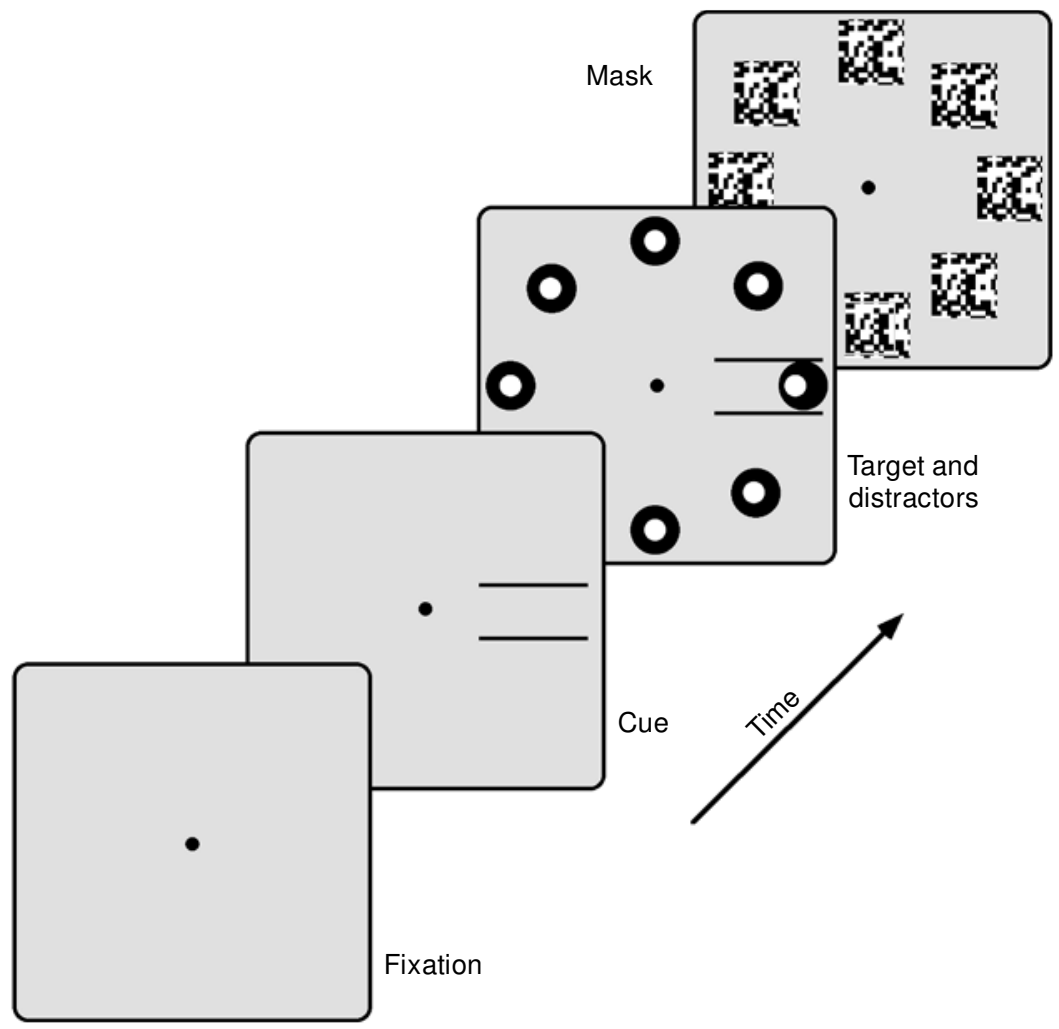

Figure 1. The general design of the experiments reported in this article. A trial started with the appearance of a fixation point for a variable period of time. Next, the cue, a pair of parallel bars, appeared, indicating where the target would appear. The target always appeared on either the left or the right side of the cue. The target and the distractors appeared next, with the cue still visible, followed by a random-dot mask in the location of each of the display items. The presentation times of the cue (cue lead time) and target (target time) in each experiment are given in the figures that present the experimental results. The cue lead and target exposure times used in the experiments were all within the time period in which transient attention operates (see Kristjánsson, Mackeben, \& Nakayama, 2001; Nakayama \& Mackeben, 1989). Note that the items are not drawn to the exact scale used in the experiment.

saccadic eye movements to the cued location (see, e.g., Leigh \& Zee, 1999). The cue stayed visible for the duration of the target exposure. The observers pressed a key according to whether the eyeballs were looking up, down, left, or right. A local mask followed the presentation of the target and the distractors. To prevent knowledge of the imaginary circle that the stimuli appeared on from revealing the target location on each trial, the center of the imaginary circle moved randomly in the horizontal direction from one trial to the next. The maximum distance the center of the circle could move relative to the center of the screen was $3.5^{\circ}$ in either direction. The cue and the target could appear at any location on the imaginary circle of targets and distractors on any given trial. Trials were run in blocks of 100 . All data points in the subsequent graphs represent at least 200 trials.

Participants. Four observers (Á.K. [the first author] and 3 observers who were not aware of the purpose of the experiment) participated. All had previous experience with the task. The observers were trained on the basic paradigm until they were able to perform the discrimination task at an accuracy above chance levels.

\section{Results and Discussion}

Figure 2 presents the results from Experiment 1 for 2 representative observers. The results for all 4 observers are presented in Table 1 . The data for all the observers showed the same pattern. It is clear that when there was a consistent relationship between the cue and the target such that the target consistently appeared at a certain colored part of the cue, discrimination performance was improved. When there was such a relationship, performance was much better than when the properties of the cue in no way predicted the location of the subsequent target (the random condition) or when the target alternated between the red and the green ends of the cue (the switch condition). This happened under both the consistent (red) and the consistent (green) conditions, as well as under the streak condition in which the target appeared on the same colored part of the cue for long stretches of contiguous trials.

This result is similar to our previous results (Kristjánsson et al., 2001), where a consistent location mapping between the cue and the target led to efficient deployments of transient attention. The present results, then, serve as a further example of the adaptability of transient attention shifts, this time ones based on color. Note that the pre- 
A
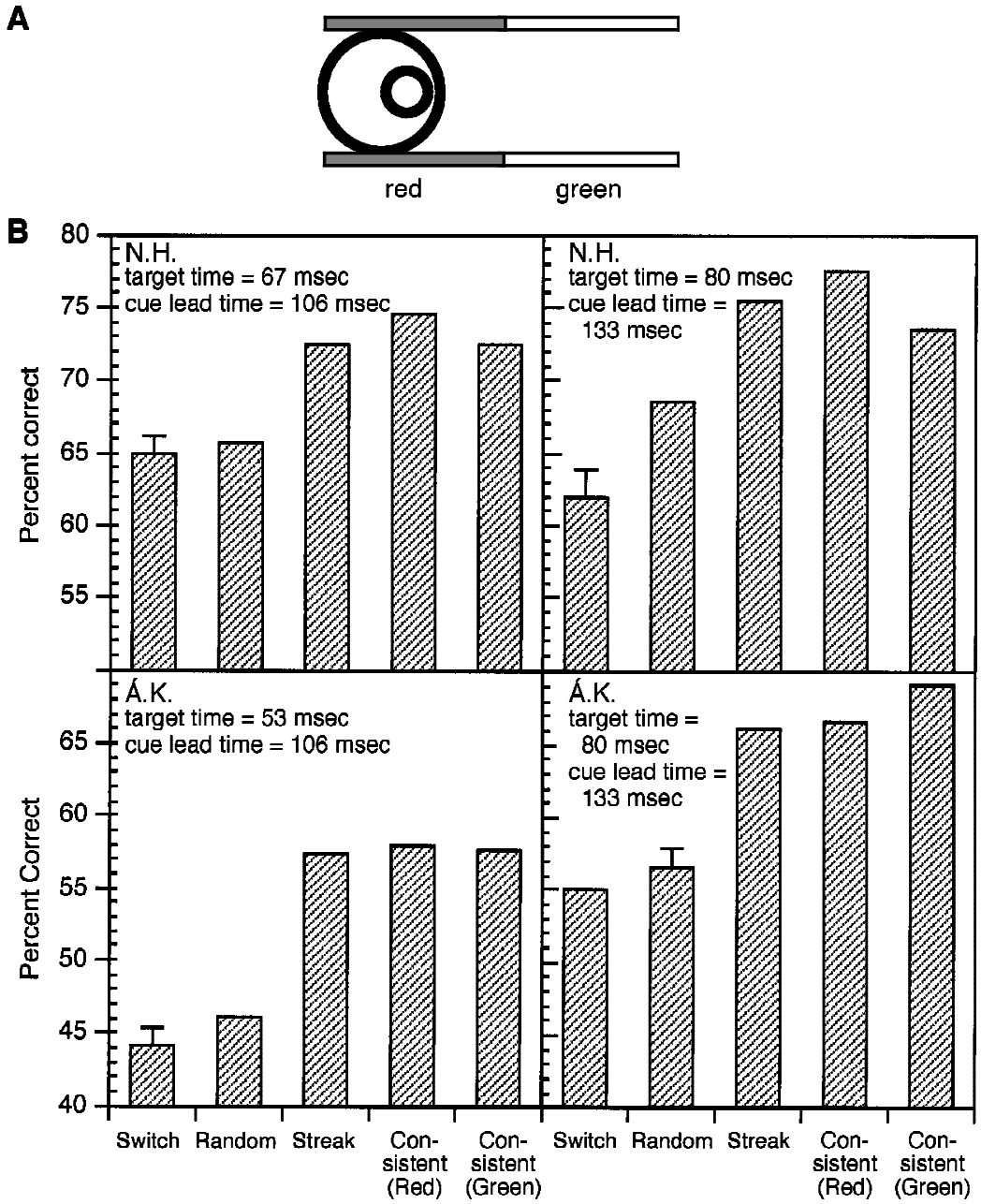

Figure 2. (A) The cue used in Experiment 1. The cue always had two colors, red on one side and green on the other. (B) The results from Experiment 1 for two combinations of cue lead and target times for 2 observers. The results for the other two were similar. The error bars show the largest standard error of proportion for each condition.

dictability of the target location from one trial to the next did not result in efficient shifts of transient attention, since performance was poor in the switch condition, in which the target location always alternated between the red and the green parts of the cue from one trial to the next. It seems, then, that transient attention tends to be allocated to the location on the cue where the target appeared on previous trials. It can thus learn feature-based relations between cue and target but seems not to be able to learn more complex relations where the target alternates between the two colors from one trial to the next (the switch condition).

The time course of the learning of the cue-target relationship was short in this experiment. Figure 3 presents the results of the streak condition as a function of where in a streak a particular trial was. On the fourth to sixth trials within a streak, performance seemed to approach a maximum (on the fourth trial within a streak, the target had appeared on the same-colored side of the cue on the last three trials). Thus, the time course of the adaptation in this experiment was similar to the time course of adaptation we observed under the consistent location mapping between the cue and the target in Kristjánsson et al. (2001).

\section{EXPERIMENT 2 \\ Shape Differences on a Cue Are Effective for the Deployment of Transient Attention}

Experiment 2 was similar to Experiment 1, except that one end of the cue differed from the other in its shape, rather than in its color (see Figure 4A). The cue was a keyholeshaped object. The target appeared on either the rounded (smooth) or the cornered (sharp) part of the cue. The purpose was to investigate whether learning similar to that observed with color in Experiment 1 would take place when 
Table 1

The Results From Experiment 1 for All 4 Observers (Percentage Correct for Each Condition)

\begin{tabular}{lccccc}
\hline & \multicolumn{5}{c}{ Condition } \\
\cline { 2 - 6 } \multicolumn{1}{c}{ Subject } & Switch & Random & Streak & $\begin{array}{c}\text { Consistent } \\
\text { (Red) }\end{array}$ & $\begin{array}{c}\text { Consistent } \\
\text { (Green) }\end{array}$ \\
\hline N.H. (67 msec, $106 \mathrm{msec})^{*}$ & 65.0 & 65.9 & 72.6 & 74.3 & 72.6 \\
N.H. (80 msec, 133 msec) & 61.9 & 68.3 & 75.2 & 77.3 & 73.1 \\
Á.K. (53 msec, 106 msec) & 44.1 & 46.1 & 57.2 & 58.1 & 57.7 \\
Á.K. (80 msec, 133 msec) & 54.9 & 56.4 & 66.0 & 66.7 & 68.8 \\
A.M.H. (67 msec, $80 \mathrm{msec})$ & 51.3 & 50.6 & 59.4 & 63.8 & 64.3 \\
A.M.H. $(80 \mathrm{msec}, 106 \mathrm{msec})$ & 60.8 & 57.3 & 68.9 & 64.7 & 70.4 \\
S.M. (67 msec, 106 msec) & 40.3 & 42.6 & 53.6 & 55.8 & 56.1 \\
S.M. (80 msec, 133 msec) & 46.8 & 45.9 & 60.1 & 59.6 & 56.4 \\
\hline
\end{tabular}

*The numbers within the parentheses denote the target appearance time and cue lead time, respectively.

the cue-target relationship depended on shape. If it is true that transient attention can learn feature-based relationships, we might observe improvement in performance as the target appeared repeatedly in the same-shaped region of the cue.

\section{Method}

As in Experiment 1, we tested five different conditions. In the switch condition, the target alternately appeared at the smooth or the sharp end of the cue from one trial to the next. In the random condition, the target appeared randomly at either end of the cue. In the streak condition, the target appeared at the same-shaped part of the cue for long stretches of contiguous trials. In the consistent (smooth) and consistent (sharp) conditions, the target always appeared at the smooth or the sharp end of the cue within each block of trials. Which part of the cue (the right or the left part) was the sharp end and which was the smooth end was determined randomly on each trial.

In general, the method was the same as that in Experiment 1, except for the different green $\left(11.8 \mathrm{~cd} / \mathrm{m}^{2}\right)$ shape cue (see Figure 4A). One combination of cue lead and target times was tested for each observer (see Figure 4B). Four observers participated in this experiment-3 naive ones, along with Á.K., one of the authors.

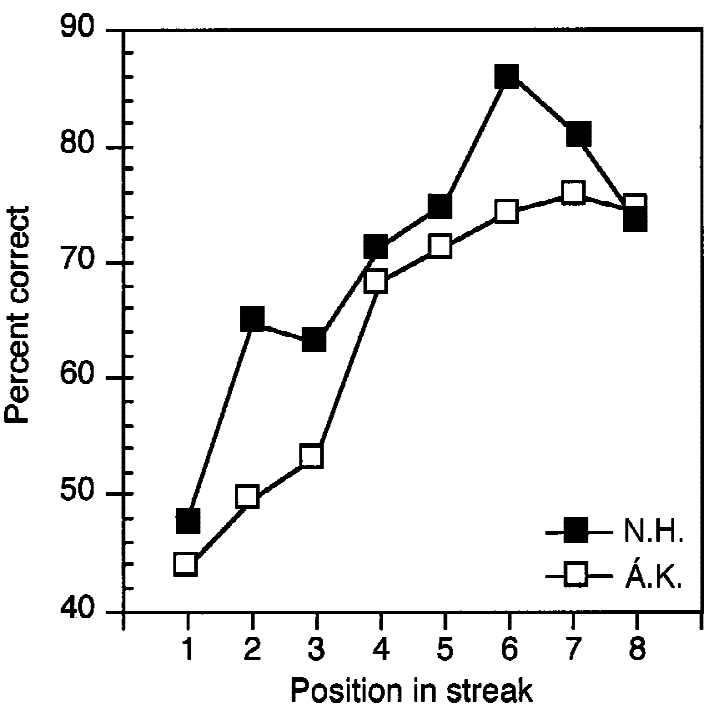

Figure 3. Discrimination performance for 2 observers in Experiment 1 as a function of where in a streak (see the text) a particular trial was located.

\section{Results and Discussion}

Shape differences on a cue are an effective stimulus for efficient transient attention shifts. Performance on the discrimination task was much better when the target consistently appeared on the same-shaped end of the cue than when there was no such relationship (see Figure 4B for the results for 2 representative observers; Table 2 presents the results for all 4 observers). Discrimination was better under both consistent conditions and for the streak condition than it was for the random and the switch conditions. Figure 5, which plots performance under the streak condition as a function of position in the streak, shows that the time course of the learning of the cue-target relationship was similar to that observed for color (Experiment 1) and location (Kristjánsson et al., 2001) cues.

Performance was somewhat better when the target appeared at the smooth end of the cue than when it appeared at the sharp end for all the observers. It is possible that the shape of the cue caused more lateral masking on the sharp side than on the smooth side. The main conclusion from Experiment 2, however, is that transient attention shows an adaptation to consistencies, in the cue-target relationship, that are based on shape that is similar to that for consistencies based on location and color. This further supports our view that transient attention can learn to go repeatedly to locations that share a certain feature but that more complex relations are beyond its capabilities, since performance in the switch condition was particularly poor, even though there was a consistent cue-target relationship.

Again, this result is not consistent with the view that transient attention is necessarily applied in a bottom-up stimulus-driven manner to abruptly appearing stimuli, supporting the conclusions of Kristjánsson et al. (2001) and the results of Experiment 1 in this article.

\section{EXPERIMENT 3 \\ A Higher Order Relationship Based on Color Is Not Effective as a Cue for Efficient Transient Attention Shifts}

An interesting aspect of the results of Experiments 1 and 2, presented here, and the results of Experiment 2 in our previous report (Kristjánsson et al., 2001) is that in the 
A
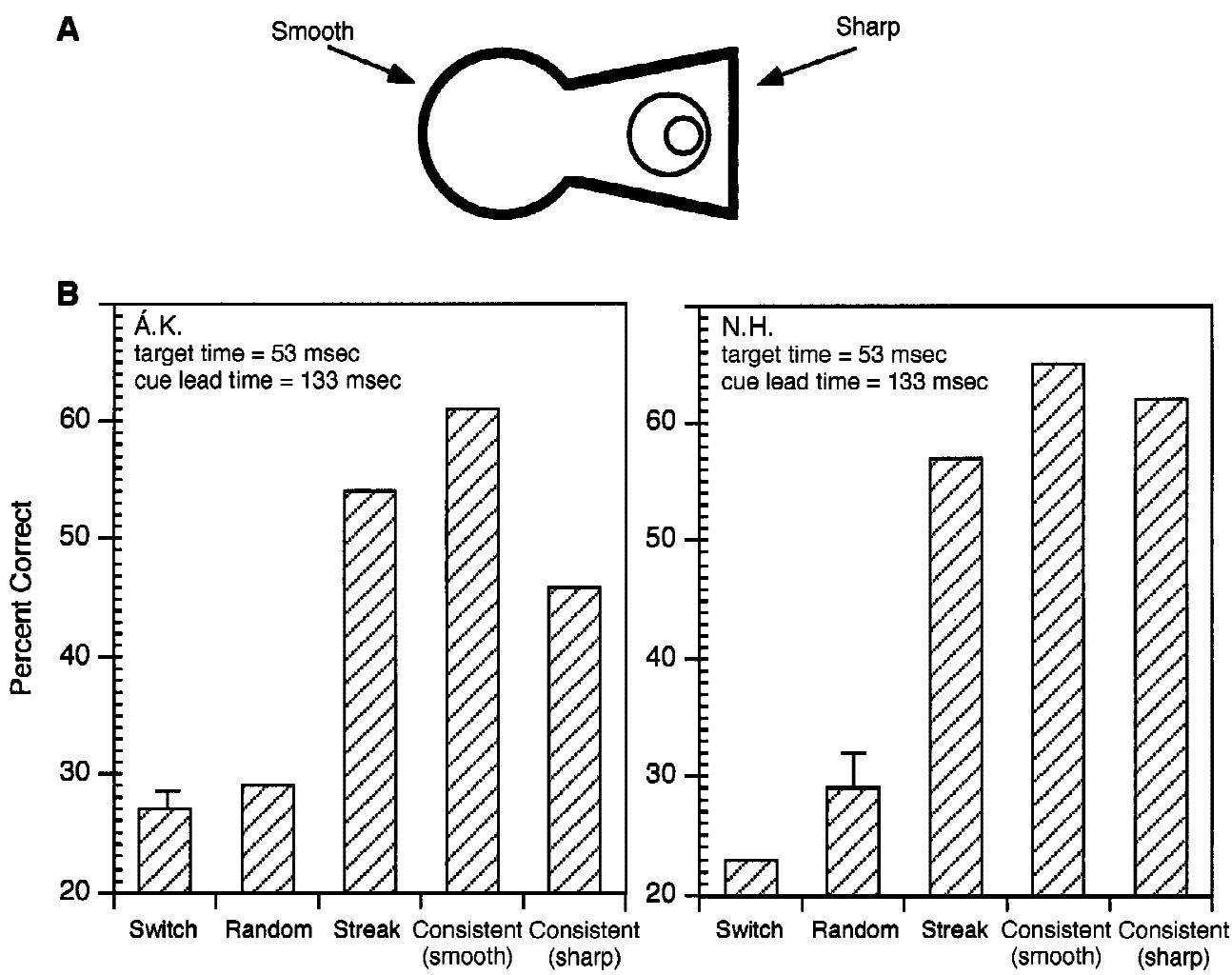

Figure 4. (A) The cue used in Experiment 2. (B) Representative results from Experiment 2 for 2 observers. The error bars show the largest standard error of proportion for each condition.

switch condition of each experiment, performance was particularly poor. The performance was, in fact, often worse than when the relationship between the cue and the target was random. This is perhaps surprising, since the target was actually in a predictable location from one trial to the next. As an example, in the switch condition of Experiment 1 , if the target appeared on the red side of the cue on the last trial, it would appear on the green side of the cue on the next trial, and so on. This sort of consistent relationship appears, however, not to lead to efficient shifts of transient attention. One possible reason is that this sort of relationship requires a two-step inference: If the target was on the green side of the cue on the last trial, then it will appear on the red side on the next. On the other hand, the re- lationships between the cue and the target that transient attention seems to be able to learn are simpler: Some feature of the cue signifies the likely target location. In Experiment 1, it was color (e.g., the target appeared on the red half of the cue); in Experiment 2, it was shape (the target appeared, e.g., on the smooth half of the cue); and in our previous paper (Kristjánsson et al., 2001), it was location (the target appeared, e.g., on the right side of the cue). No higher order inference is thus required to take advantage of consistencies of this sort (a one-step inference is sufficient).

If the above is actually the explanation for the poor performance under the switch condition of the previous experiments, it might be possible to address that experimen-

Table 2

The Results From Experiment 2 for All 4 Observers (Percentage Correct for Each Condition)

\begin{tabular}{lccccc}
\hline & \multicolumn{5}{c}{ Condition } \\
\cline { 2 - 6 } \multicolumn{1}{c}{ Subject } & Switch & Random & Streak & $\begin{array}{c}\text { Consistent } \\
\text { (Smooth) }\end{array}$ & $\begin{array}{c}\text { Consistent } \\
\text { (Sharp) }\end{array}$ \\
\hline Á.K. (53 msec, $133 \mathrm{msec}$ )* & 27.2 & 29.1 & 54.2 & 60.3 & 45.6 \\
N.H. (53 msec, 133 msec) & 23.0 & 28.2 & 56.8 & 65.0 & 61.5 \\
A.M.H. (53 msec, $133 \mathrm{msec})$ & 33.6 & 38.4 & 58.3 & 64.6 & 62.8 \\
A.H. (53 msec, $133 \mathrm{msec})$ & 28.6 & 30.9 & 53.3 & 58.6 & 55.8 \\
\hline
\end{tabular}

*The numbers within the parentheses denote the target appearance time and cue lead time, respectively. 


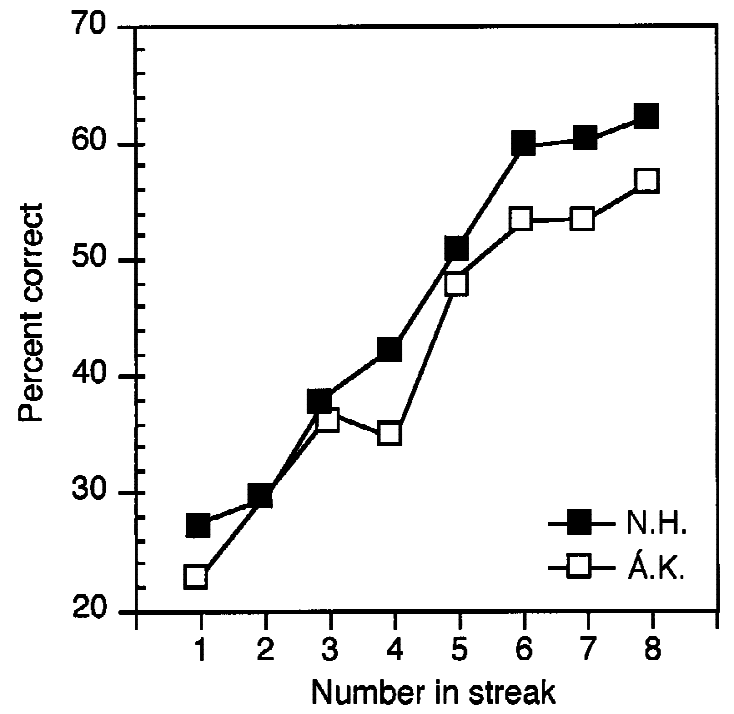

Figure 5. Discrimination performance for 2 representative observers in Experiment 2 as a function of where in a streak (see the text) a particular trial was located.

tally. Experiment 3 tested performance when a higher order inference was required to uncover the consistent relationship between the cue and the target. The cue was of one color on each trial, either red or green, picked randomly from one trial to the next. In the consistent condition, the location of the target depended on the color of the cue; thus, if the cue was red, the target appeared on the right side of the cue, whereas it appeared on the left side if the cue was green. To take advantage of the consistent relationship between the cue and the target, a two-step inference was required: If the cue is of a certain color, then the target will be at a certain location.

\section{Method}

The cue was a pair of parallel green $\left(11.8 \mathrm{~cd} / \mathrm{m}^{2}\right)$ or $\mathrm{red}\left(9.7 \mathrm{~cd} / \mathrm{m}^{2}\right)$ bars. In the consistent condition, the target always appeared at a fixed location on the cue on a given trial, depending on the color of the cue. So, for example, if the target appeared on the right side of the red cue, it would appear on the left side of the green cue, and that pattern would continue within that particular block of trials. In contrast, in the random condition, the target appeared randomly at either end of the cue, irrespective of its color. The target could appear only on the left or the right side of the cue.

Two naive observers, along with Á.K. (one of the authors), participated in the experiment. Two different combinations of cue lead and target times were tested in this experiment (see Figure 6B). The method was in other respects similar to that described for Experiment 1 in this article.

\section{Results and Discussion}

Figure 6B shows the results for 2 representative observers, whereas Table 3 shows the results for all 3 observers that were tested. It is clear that the observers were not able to learn the relationship between the cue and the target during shifts of transient attention in the consistent condition of this experiment. Discrimination performance was similar under the consistent and the random conditions under both combinations of cue lead and target times for both observers.

For transient attention to adapt to the relationship between the cue and the target in the consistent condition of Experiment 3, a two-step inference was required. Such a relationship was, according to our results, not sufficient for learning, similar to the learning we observed in Experiments 1 and 2, to take place. This finding, coupled with the results of the switch conditions of Experiments 1 and 2, suggests that transient attention cannot adapt to higher order relationships of this sort.

Figure 7 conveys an interesting aspect of the results. There, we have plotted performance under the consistent condition of Experiment 3 as a function of how often in a row the target appeared in the same location on the cue. The more often the target appeared in the same location on the cue, the more performance improved. This result echoes our findings from Experiment 2 in Kristjánsson et al. (2001), in which a consistent location mapping between cue and target resulted in rapid learning of the cue-target relationship. Thus, even though there was a consistent relationship, between the cue and the target, based on color in this experiment, the consistency in target location relative to the cue that was due to random fluctuations in consistency resulted in efficient shifts of transient attention, perhaps effectively overriding the color consistency.

\section{EXPERIMENT 4 \\ A Higher Order Relationship Based on Shape is Not an Effective Cue for Efficient Shifts of Transient Attention}

In Experiment 4, we investigated whether a relationship between cue and target similar to that tested in Experiment 3 , one based on shape, would result in efficient shifts of transient attention. We used two cues of different shapes in this experiment (see Figure 8a). In the consistent condition, the target appeared consistently at only one end of each cue, whereas in the random condition, the target appeared randomly at either end of each cue. So, in the consistent condition, if the smooth cue was presented, the target would always appear at its left end, for example, within a block of trials, whereas it would appear at the right end of the sharp cue. In the random condition, there was no such consistency, so the cue could appear at either end of the two different cues.

\section{Method}

Three observers, Á.K. (the first author) and 2 experienced observers who were unaware of the goals of the experiment, participated. In all other respects, the method was similar to those in the previous experiments of this paper, described above.

\section{Results and Discussion}

The results from Experiment 4, presented in Figure 8b (for 2 representative observers), were similar to the results of Experiment 3 (Table 4 presents the results for all $3 \mathrm{ob}-$ 
A

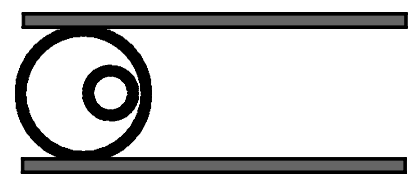

red



green

B

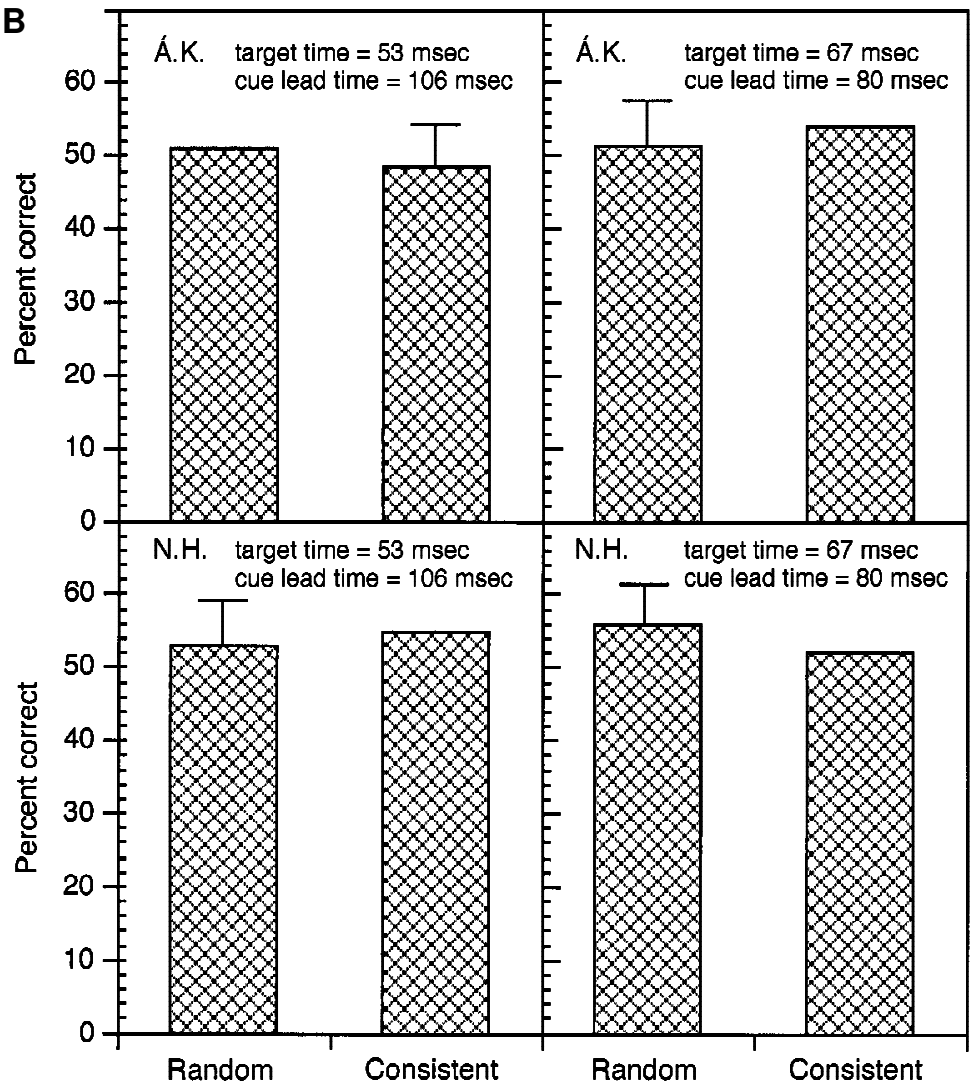

Figure 6. The results of Experiment 3. The figure shows discrimination performance for 2 observers under two different combinations of cue lead and target times. The error bars show the largest standard error of proportion for each condition.

servers). A higher order relationship between the cue and the target, one based on shape, did not result in efficient shifts of transient attention. Performance under the consistent condition was no better than when the shape of the cue in no way predicted the location of the target within it (the random condition).

Figure 9 plots the performance under the consistent condition of Experiment 4 as a function of how often in a row the target appeared in the same location on the cue. As in Experiment 3, we found that accidental consistent location mapping of cue and target resulted in efficient shifts of transient attention. The more often the target appeared at the same location, relative to the cue, in succession, the better the performance.

From the results, it is clear that transient attention ignores, or is unable to utilize, higher order relationships between cue and target, at least when an if-then inference is required to uncover the relationship. On the other hand, simple relationships, such as a consistent location mapping (Kristjánsson et al., 2001) or feature-based relationships (Experiments 1 and 2 in this article), are very effective for focused deployment, even overriding other, seemingly

Table 3

The Results From Experiment 3 for All 3 Observers (Percentage Correct for Each Condition)

\begin{tabular}{lcc}
\hline \multirow{2}{*}{ Subject } & \multicolumn{2}{c}{ Condition } \\
\cline { 2 - 3 } & Random & Consistent \\
\hline Á.K. (53 msec, $106 \mathrm{msec})^{*}$ & 50.5 & 49.1 \\
Á.K. (67 msec, $80 \mathrm{msec})$ & 50.7 & 51.6 \\
N.H. $(53 \mathrm{msec}, 106 \mathrm{msec})$ & 51.8 & 52.4 \\
N.H. $(67 \mathrm{msec}, 80 \mathrm{msec})$ & 53.2 & 50.9 \\
A.M.H. $(53 \mathrm{msec}, 106 \mathrm{msec})$ & 49.6 & 51.3 \\
A.M.H. $(67 \mathrm{msec}, 80 \mathrm{msec})$ & 52.8 & 50.9 \\
\hline
\end{tabular}

*The numbers within the parentheses denote the target appearance time and cue lead time, respectively. 
more informative cues to improve discrimination performance (as is shown in Figure 9).

\section{EXPERIMENT 5 Explicit or Implicit Learning?}

In the experiments described so far, the cue lead and target times tested were very brief. This makes us confident that we were testing the properties of transient shifts of visual attention, attention shifts that do not seem to be dependent on explicit top-down control (see Kristjánsson et al., 2001; Nakayama \& Mackeben, 1989). One can ask, however, what role explicit knowledge of the cue-target relationship plays in the learning we found. In other words, can the learning that we observed be attributed to topdown strategies in any way? Our naive observers were never informed of any differences between the different conditions of each experiment. It is, however, possible that they may have "picked up" on the cue-target relationship and used that to aid their performance. To assess what effect explicit knowledge of the relationship between the cue and the target plays in our experiments, we ran a study in which the naive observers were informed of the cuetarget relationship and were asked to try to utilize this information to aid their performance. We tested this both for cue-target relationships that transient attention could learn (from Experiment 1) and for relationships that were not amenable to this form of learning (from Experiment 3).

\section{Method}

The method was identical to those described for Experiments 1 and 3 , except that the 2 observers were told explicitly about the cue-target relationship in each case and were told to try to use that information to perform the task. The cue had either two colors (red and green), as in Experiment 1, or one color, as in Experiment 3. In the switch/two-color condition, the observers were told that the target would switch between appearing on the green side and on the red side of the cue from one trial to the next. In the streak/two-color condition, they were told that the target would most likely appear on the same-colored side of the cue for long stretches of adjacent trials. In the random/one-color condition, they were told that there was no consistent relationship between the color of the cue and the location of the target, whereas in the consistent/one-color condition, they were told that if the cue was red, the target would appear on the right side of the cue and on the left side if the cue was green. The cue lead time was $140 \mathrm{msec}$, and the target exposure time was $60 \mathrm{msec}$.

\section{Results and Discussion}

The results from the fifth experiment for the 2 observers are shown in Figure 10. It is clear that the observers were not able to utilize their knowledge of the cue-target relationship to aid their performance. In both conditions in which the cue was of one color, performance was similar (Figure 10A), even though the observers knew of the connection between the target and the cue in the consistent condition and, also, there was no such consistent relation in the random condition. On the other hand, performance was quite good in the streak/two-color condition with a cue of two colors and a lot worse in the switch condition with the two-colored cue (see Figure 10B). Thus, perfor-



Figure 7. The accidental streaks of Experiment 3 (see the text).

mance was the same as in Experiments 1 and 3 even though the observers had explicit knowledge of the cue-target contingency in each case. Furthermore, the data show a learning effect within a streak similar to that observed in Experiment 1 (Figure 10C). The main conclusion we draw from this is that the learning we observed in Experiments 1 and 2 was independent of whether the observers had explicit knowledge of the cue-target relationship. In other words, they could not use top-down knowledge to perform this task. This is perhaps not surprising if one considers how brief the cue $(140 \mathrm{msec})$ and the target $(60 \mathrm{msec})$ presentation times were. Ward, Duncan, and Shapiro (1996) found that when attention was grabbed by a stimulus, the attentional switching time to another location was quite long, possibly up to $500 \mathrm{msec}$. Thus, it is probably not surprising that a volitional attention deployment cannot take place in less than $200 \mathrm{msec}$. A more reflexive mechanism must be relied on to perform tasks such as this.

The fact that the observers could not apply their explicit knowledge of cue-target relations to this task is reminiscent of Maljkovic and Nakayama's (1994) finding that observers could not willfully overcome the priming effects they found in their priming of pop-out paradigm. In fact, when our observers were asked, after the experiment, whether they had been able to use the information they were given on cue-target relations to aid their performance, they unanimously reported that it was impossible. The presentation times were simply too brief.

\section{EXPERIMENT 6 Performance is the Same Before and After Briefing}

Although the results from Experiment 5 indicate that no top-down strategies can be applied to the performance of this task, we would be more confident in drawing that 
A

Sharp

Smooth


Figure 8. (A) The two different cues used in Experiment 4. (B) The results from Experiment 4 for two combinations of cue lead and target times for 2 of the observers. The error bars show the largest standard error of proportion for each condition.

conclusion if we could get a similar pattern of performance for naive observers before and after explicit instruction about the cue-target relationship. In Experiment 6, we tested 3 naive observers on the same conditions as those in Experiment 5, before and after being informed about the exact nature of the cue-target relationship.

Furthermore, we added a verbal cue below the fixation point on the screen after the briefing, which indicated on which side of the cue the target would appear on the following trial. If only minor differences were to be found between performance with or without information about the cue-target contingencies, it would strengthen our conclusion that explicit top-down strategies cannot be used in this task, since we would get a direct comparison of performance before and after observers were briefed about the cue-target relationship. In addition, we asked our observers, after the first part of the experiment (when no instruction was given), whether they had noticed any pat- terns in the cue-target relationships under the different conditions.

\section{Method}

Each observer participated twice in each of the four conditions of the experiment (the switch/two-color, streak/ two-color, random/

Table 4

The Results From Experiment 4 for all 3 Observers (Percentage Correct for Each Condition)

\begin{tabular}{lcc}
\hline & \multicolumn{2}{c}{ Condition } \\
\cline { 2 - 3 } Subject & Random & Consistent \\
\hline Á.K. (53 msec, $106 \mathrm{msec})^{*}$ & 44.6 & 46.2 \\
Á.K. (67 msec, $80 \mathrm{msec})$ & 51.9 & 49.8 \\
A.M.H. (53 msec, $106 \mathrm{msec})$ & 49.9 & 48.4 \\
A.M.H. (67 msec, $80 \mathrm{msec})$ & 50.7 & 50.3 \\
W.S. (53 msec, $106 \mathrm{msec})$ & 53.8 & 54.6 \\
W.S. (67 msec, $80 \mathrm{msec})$ & 50.3 & 51.2 \\
\hline
\end{tabular}

*The numbers within the parentheses denote the target appearance time and cue lead time, respectively. 


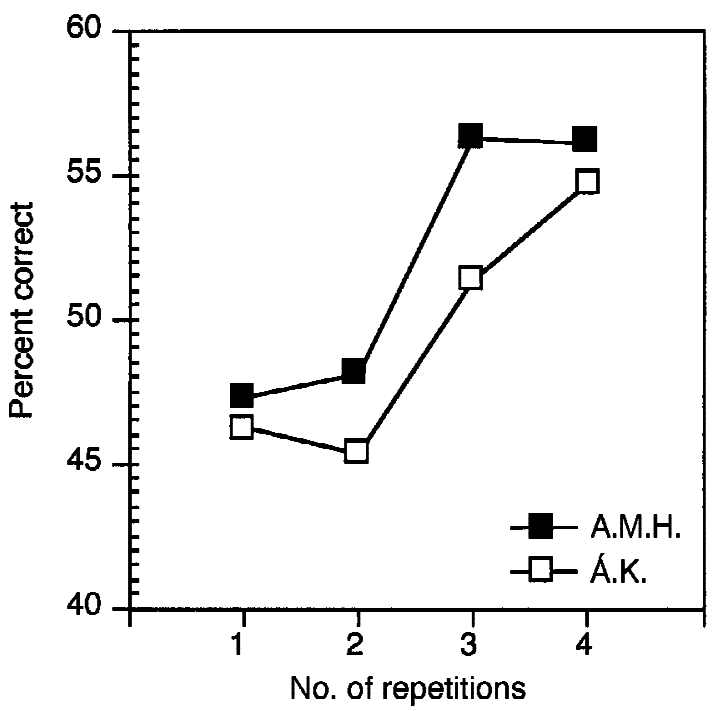

Figure 9. The accidental streaks of Experiment 4 (see the text).

one-color, and consistent/one-color conditions; see the Method section of Experiment 5) - once without receiving any information about the different conditions (they were told only that the cue would reliably indicate the target location) and once after being informed about the exact nature of the cue-target relationship under each condition. After performing the no-instruction part of the experiment, they were asked whether they had noted any systematic relation between the properties of the cue and the target location. Then, after performing the full-instruction part, they were asked whether they felt that the information about the cue-target contingency had aided their performance.

In the full-instruction condition, we presented a verbal cue (RIGHT or LEFT) on the screen right below the fixation point, which indicated whether the target would appear on the right or the left side of the cue on the following trial. When the color cue appeared, the verbal cue disappeared. In other respects, the method was similar to that described for Experiment 5 above.

\section{Results and Discussion}

The results from Experiment 6 are shown in Figure 11 as differences in discrimination performance before and after the instruction about the cue-target relationship. There are no significant differences between performance before and after the observers were told about the cue-target contingency (chi-square tests, $d f=1, p>.3$, for all cases). None of the 3 observers reported noticing any differences between the conditions after the no-instruction part of the experiment, and all the observers said that it was quite impossible to use their knowledge of the cue- target relation, or the verbal cue on the screen, after they had been informed about it (in the full-instruction condition). They reported that the exposure times were simply too brief. This result reinforces our conclusions that explicit knowledge of the contingencies between the cue and the target cannot be used to aid performance in the tasks used in the set of experiments reported here. Thus, we conclude that the shortterm learning system mediating the learning we have observed is an autonomous learning mechanism not under top-down control. This result is reminiscent of the results of Lambert and colleagues (Lambert, Naikar, McLachlan, \& Aitken, 1999; Lambert, Norris, Naikar, \& Aitken, 2000; Lambert \& Sumich, 1996) on so-called implicit peripheral cuing, where visual orienting was influenced by peripheral letter cues, even though observers were completely unaware of the validity of the cues.

\section{GENERAL DISCUSSION}

In this article, we have shown that when transient visual attention is shifted to an abruptly appearing stimulus, the deployment can be quite focused, depending on where a behaviorally relevant stimulus has previously appeared relative to that object. The results extend our previous findings (Kristjánsson et al., 2001) to domains other than just location consistency; they show that such relationships based on shape and color can also lead to efficient shifts of transient attention. Previous experiments indicating that transient attention is reflex-like and indiscriminately applied over suddenly appearing stimuli did not reveal these benefits, perhaps because they relied on reaction time measures of the detection of abruptly appearing stimuli, whereas our results indicate that these efficient shifts


Figure 10. The results of the explicit instruction experiment (Experiment 5) for 2 representative observers (see the text for details). 


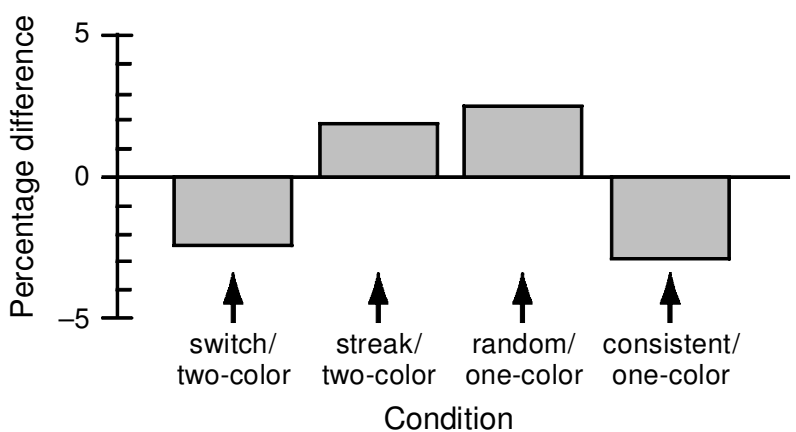

Figure 11. The results of Experiment 6 (see the text for details). The graph shows the differences in percentages of correct responses before (no instruction) and after (full instruction) briefing on the cue-target contingency.

of attention can improve performance on a discrimination task. Furthermore, the results from Experiments 3 and 4 serve a useful purpose, since they show the limits of learning of this sort. A higher order relationship between the cue and the target does not result in efficient transient attention shifts, ${ }^{2}$ indicating that this is a primitive and very specialized form of learning. ${ }^{3}$ Experiments 5 and 6 , then, show that top-down knowledge of the contingency between the cue and the target has no effect on performance. Even though the observers had explicit knowledge about the relationships and tried, furthermore, to use that knowledge to aid their performance, the results were the same as those in the previous experiments, in which the naive observers were not aware of the different cue-target contingencies. ${ }^{4}$

The results are seemingly in harmony with the idea that top-down strategies can affect how likely abruptly onset stimuli are to capture attention (see, e.g., Bacon \& Egeth, 1994; Folk \& Remington, 1999; Folk et al., 1992; Gibson \& Jiang, 1998). There is, however, one important difference between the learning exhibited here and these topdown effects on attentional capture: Experiments 5 and 6 in this article show that this form of learning is independent of whether observers are aware of the cue-target relationship or not. Our findings, then, cannot be considered simply an example of top-down knowledge's overriding stimulus-driven attentional capture. The learning, then, seems to be more implicit than explicit and not under topdown control, which distinguishes it from research showing top-down modulation of attentional capture. It is, in fact, quite possible that the top-down modulation of capture observed in the research on contingent capture (Folk \& Remington, 1999; Folk et al., 1992) is partly mediated by the learning mechanisms investigated here. 5

\section{Potential Benefits}

What are the potential benefits of such a learning mechanism? Outside the laboratory and, perhaps, the video game arcade, this form of learning in transient attention shifts may allow fast orienting to the relevant features of a potential predator or, perhaps, toward parts of suddenly appearing moving objects that give the most information about the objects' potential trajectory. The learning could thus benefit organisms in that it allows faster responses to danger. It is, however, important in this context to note the limitations of this form of learning, as revealed in Experiments 3 and 4. This learning mechanism can redirect attention to previously attended features and locations but seems not to be able to make predictions about locations of important stimuli on the basis of the characteristics of a preceding stimulus.

\section{Possible Implications for Visual Memory and Visual Representations}

Recent research has demonstrated that visual memory can often be surprisingly poor and prone to errors (Rensink, O'Regan, \& Clark, 1997; Simons \& Levin, 1997; Wolfe, 1999). A typical finding from such change blindness studies is that observers are surprisingly inept at spotting seemingly important changes to their visual environment. The apparent stability of our visual representation of the environment may thus be somewhat illusory. Rensink (2000; see also Ballard, Hayhoe, Pook, \& Rao, 1997; O'Regan, 1992) hypothesized that instead of maintaining an accurate representation of every detail in the visual environment, the visual system forms representations as needed, "just in time" for their use. The results of our experiments in this article and the companion paper (Kristjánsson et al., 2001) may uncover one way the visual system forms these fleeting representations. What seems clear is that transient attention is deployed to where a task-relevant stimulus has been found in the immediate past, which may be a way of quickly reforming previously constructed representations. Priming of pop-out (Kristjánsson, Wang, \& Nakayama, 2002; Maljkovic \& Nakayama, 1994, 1996), contextual cuing (Chun \& Jiang, 1999), and maybe even inhibition of return (Klein, 2000; Posner \& Cohen, 1984) could be other ways of achieving similar goals (see also Chun \& Nakayama, 2000; Kristjánsson, 2000).

If some aspect of a search display recently seen can easily be relocated in a new display, there should be a benefit to that, insofar as a previous behavior is to be repeated. Environments that organisms encounter do not change randomly from one moment to the next; instead, fundamental aspects of those surroundings are stable over time. Quick orientation to stimuli that takes into account previous encounters with a similar stimulus could thus be very beneficial.

\section{Possible Neural Correlates}

A possible candidate for the neural correlates of the learning uncovered in the experiments presented in this paper is activity in the supplementary eye fields (SEFs) in the prefrontal cortex. Evidence from single-cell recordings of the SEFs suggests that they play a role in eye movement preparation in object-based coordinates, rather than in coordinates based on absolute position (Chen \& Wise, 1995). Chen and Wise also suggested that the SEFs are part of a neural system "learning flexible, non-spatial stimulus-response relations" (p. 1101). Further evidence 
for object-centered response properties of neurons in the SEFs comes from single-cell recordings by Olson and Gettner $(1995,1996,1999)$. They found SEF neurons that were activated most strongly when the animals were preparing saccadic eye movements to particular locations on a stimulus, irrespective of its absolute position. The important point here is that the SEFs show learning of spatial relations between two stimuli in object-centered, rather than "absolute" position-centered, coordinates. This form of learning is exactly what our experiments indicate that transient attention is capable of, since the learning we observed was object based - that is, it happened irrespective of the absolute position of the cue and the target. Whether neural units in the SEFs are indeed capable of this form of learning remains to be seen, but the possibility remains, in light of the neurophysiological findings and the fact that many studies have shown a tight link between saccadic eye movements and shifts of visual attention, both at the neural level (Kustov \& Robinson, 1996) and behaviorally (Deubel \& Schneider, 1996; Hoffman \& Subramaniam, 1995; Kowler, Anderson, Dosher, \& Blaser, 1995). More concretely, then, it is possible that neurons in the SEFs would show a modulation of their responses that is correlated with learning in the deployment of transient attention.

\section{Relation to Eye Movements}

There is good reason to think that the form of learning uncovered with the experiments in this article is related to eye movement preparation. First, many studies have uncovered a strong link between shifts of visual attention and saccadic eye movements (Deubel \& Schneider, 1996; Hoffman \& Subramaniam, 1995; Kowler et al., 1995; McPeek, Maljkovic, \& Nakayama, 1999). Second, if, as was proposed above, the learning that we observed is at least in part generated by activity in the SEFs, this would immediately suggest a link between the shifts of attention investigated here and eye movement preparation, given the role played by the SEFs in eye movements and especially, purposeful eye movements in object-centered coordinates (Olson \& Gettner, 1995; see also Schall, 1991). Insofar as attentional deployments precede eye movements, there is a possibility that consistencies like the ones studied in this article would lead not only to more efficient attention shifts, but also to faster, or more accurate, eye movements to targets of this sort, targets that have a consistent relationship with another stimulus. In fact, recent results from our laboratory indicate that this may indeed be the case (Edelman, Kristjánsson, \& Nakayama, 2001; see also Edelman, Cherkasova, \& Nakayama, 2002).

\section{Conclusions}

The learning of the relationship between the cue and the target that was revealed in these experiments suggests the existence of a mechanism for the deployment of attention that takes into account previous appearances of the behaviorally relevant stimuli. This mechanism is not under volitional control and seems to operate in object-based, rather than space-based or retinotopic, coordinates. This learning mechanism is potentially useful in allowing fast reorienting to previously encountered objects, as well as quick reconstruction of previously viewed objects.

\section{REFERENCES}

BACon, W. F., \& EgETH, H. E. (1994). Overriding stimulus-driven attentional capture. Perception \& Psychophysics, 55, 485-496.

Ballard, D. H., Hay hoe, M. M., Pook, P. K., \& Rao, R. P. (1997). Deictic codes for the embodiment of cognition. Behavioral \& Brain Sciences, 20, 723-767.

BReITMEYER, B. G., \& GANZ, L. (1976). Implications of sustained and transient channels for theories of visual pattern masking, saccadic suppression and information processing. Psychological Review, 83, 1-36.

Cheal, M. L., \& Lyon, D. R. (1991). Central and peripheral precuing of forced-choice discrimination. Quarterly Journal of Experimental Psychology, 43A, 719-746.

Chen, L. L., \& Wise, S. P. (1995). Neuronal activity in the supplementary eye field during acquisition of conditional oculomotor associations. Journal of Physiology, 73, 1101-1121.

Chun, M. M., \& JiAng, Y. (1999). Top-down attentional guidance based on implicit learning of visual covariation. Psychological Science, 10, 360-365.

Chun, M. M., \& Nakayama, K. (2000). On the functional role of implicit visual memory for the adaptive deployment of attention across scenes. Visual Cognition, 7, 65-81.

Deubel, H., \& Schneider, W. X. (1996). Saccade target selection and object recognition: Evidence for a common attentional mechanism. Vision Research, 36, 441-452.

Edelman, J. A., Cherkasova, M. V., \& Nakayama, K. (2002). A spatial memory system for the guidance of eye movements in crowded visual scenes [Abstract]. Journal of Vision, 2 (7), 572a. Available: http:// journalofvision.org/2/7/572.

Edelman, J. A., Kristjánsson, Á. \& Nakayama, K. (2001). Facilitation of saccade target selection by object centered priming [Abstract]. Journal of Vision, 1 (3), 238a. Available: http://journalof vision.org/ $1 / 3 / 238 /$

Folk, C. L., \& Remington, R. (1999). Can new objects override attentional control settings? Perception \& Psychophysics, 61, 727-739.

Folk, C. L., Remington, R., \& Johnston, J. C. (1992). Involuntary orienting is contingent on attentional control settings. Journal of Experimental Psychology: Human Perception \& Performance, 18, 10301044.

GiBSON, B. S., \& JiAnG, Y. (1998). Surprise! An unexpected color singleton does not capture attention in visual search. Psychological Science, 9, 176-182.

Hoffman, J. E., \& Subramaniam, B. (1995). The role of visual attention in saccadic eye movements. Perception \& Psychophysics, 57, 787-795.

JoNIDES, J. (1981). Voluntary versus automatic control over the mind's eye's movement. In J. [B.] Long \& A. [D.] Baddeley (Eds.), Attention and performance IX (pp. 187-203). Hillsdale, NJ: Erlbaum.

Kingstone, A., \& Klein, R. (1991).Combining shape and position expectancies: Hierarchical processing and selective inhibition. Journal of Experimental Psychology: Human Perception \& Performance, 17, 512-519.

KLEIN, R. M. (2000). Inhibition of return. Trends in Cognitive Sciences, 4, 138-147.

Kowler, E., Anderson, E., Dosher, B., \& Blaser, E. (1995). The role of attention in the programming of saccades. Vision Research, $\mathbf{3 5}$, 1897-1916.

KRISTJÁNSSON, Á. (2000). In search of remembrance: Evidence for a memory for locations in visual search. Psychological Science, 11, 328-332.

Kristjánsson, Á., Mackeben, M., \& Nakayama, K. (2001). Rapid learning in the deployment of transient attention. Perception, $\mathbf{3 0}$, 1375-1387.

Kristúnsson, Á., Wang, D., \& Nakayama, K. (2002). The role of priming in conjunctive visual search. Cognition, 85, 37-52.

Kustov, A. A., \& Robinson, D. L. (1996). Shared neural control of attentional shifts and eye movements. Nature, 384, 74-77. 
Lambert, A., Naikar, N., McLachlan, K., \& Aitken, V. (1999). A new component of visual orienting: Implicit effects of peripheral information and subthreshold cues on covert attention. Journal of Experimental Psychology: Human Perception \& Performance, 25, 321-340.

Lambert, A., Norris, A., Naikar, N., \& Aitken, V. (2000). Effects of informative peripheral cues on eye movements: Revisiting William James' 'derived attention.' Visual Cognition, 7, 545-569.

Lambert, A., \& Sumich, A. L. (1996). Spatial orienting controlled without awareness: A semantically based implicit learning effect. Quarterly Journal of Experimental Psychology, 49A, 490-518.

LEIGH, R. J., \& $Z_{\mathrm{EE}}, \mathrm{D}$. S. (1999). The neurology of eye movements (3rd ed.). New York: Oxford University Press.

Lennie, P. (1980). Parallel visual pathways: A review. Vision Research, 20, 561-594.

Luck, S. J., \& Thomas, S. J. (1999). What variety of attention is automatically captured by peripheral cues? Perception \& Psychophysics, 61, 1424-1435.

Maljkovic, V., \& Nakayama, K. (1994). Priming of pop-out: I. Role of features. Memory \& Cognition, 22, 657-672.

Maljkovic, V., \& Nakayama, K. (1996). Priming of pop-out: II. The role of position. Perception \& Psychophysics, 58, 977-991.

McPeek, R. M., Maljkovic, V., \& Nakay ama, K. (1999). Saccades require focal attention and are facilitated by a short-term memory system. Vision Research, 39, 1555-1566.

NAKAYAMA, K., \& MACKeben, M. (1989). Sustained and transient components of visual attention. Vision Research, 29, 1631-1647.

Olson, C. R., \& Gettner, S. N. (1995). Object-centered direction selectivity in the macaque supplementary eye field. Science, $\mathbf{2 6 9}, 985$ 988.

Olson, C. R, \& GetTner, S. N. (1996). Representation of object-centered space in the primate frontal lobe. Cognitive Brain Research, 5, 147-156.

Olson, C. R., \& GetTner, S. N. (1999). Macaque SEF neurons encode object-centered directions of eye movements regardless of the visual attributes of instructional cues. Journal of Neurophysiology, 81, 23402346.

O'REGAN, J. K. (1992). Solving the "real" mysteries of visual perception: The world as an outside memory. Canadian Journal of Psychology, 46, 461-488.

Posner, M. I., \& CoHen, Y. (1984). Components of attention. In H. Bouma \& D. G. Bouwhuis (Eds.), Attention and performance X: Control of language processes (pp. 531-556). Hillsdale, NJ: Erlbaum.

Rensink, R. A. (2000). Seeing, sensing, and scrutinizing. Vision Research, 40, 1469-1487.

Rensink, R. A., O'Regan, J. K., \& Clark, J. J. (1997). To see or not to see: The need for attention to perceive changes in scenes. Psychological Science, 8, 368-373.

Schall, J. D. (1991). Neuronal activity related to visually guided saccadic eye movements in the supplementary motor area of rhesus monkeys. Journal of Neurophysiology, 66, 530-558.

Simons, D. J., \& Levin, D. T. (1997). Change blindness. Trends in Cognitive Sciences, 1, 261-267.

TheEUWES, J. (1991). Exogenous and endogenous control of attention: The effect of visual onsets and offsets. Perception \& Psychophysics, 49, 83-90.

Theeuwes, J., \& Burger, R. (1998). Attentional control during visual search: The effect of irrelevant singletons. Journal of Experimental Psychology: Human Perception \& Performance, 24, 1342-1353.

Ward, R, Duncan, J., \& Shapiro, K. (1996). The slow time-course of visual attention. Cognitive Psychology, 30, 79-109.

Wolfe, J. M. (1999). Inattentional amnesia. In V. Coltheart (Ed.), Fleeting memories: Cognition of brief visual stimuli (pp. 71-94). Cambridge, MA: MIT Press.

YANTIS, S., \& JoNidES, J. (1984). Abrupt visual onsets and selective attention. Evidence from visual search. Journal of Experimental Psychology: Human Perception \& Performance, 10, 601-621.

YANTIS, S., \& JonideS, J. (1996). Attentional capture by abrupt onsets: New perceptual objects or visual masking? Journal of Experimental Psychology: Human Perception \& Performance, 22, 1505-1513.

\section{NOTES}

1. The streaks were generated in the following way. The probability that the target would appear on the same colored part of the cue as on the previous trial was equal to $1-\{N[0.1-(0.01 N)]\}$, where $N$ is the present number of sequential target appearances at the same colored part of the cue. Thus, if the target appeared at the red end of the cue on trial $n-1$ and on the green part of the cue on trial $n-2$, the probability that the target would appear at the red end on the present trial $(n)$ was .91, on trial $n+1$ it was .84 , and so on. The probability function was set to asymptote at the probability $=.75$ so that when $N$ was 5 , the probability was .75 and remained so until $N=8$, where the probability was set to 0 , since the maximum length of a streak was eight.

2.We cannot, of course, rule out that such relationships could be found in future research, as was pointed out by one of our reviewers.

3 . We can rule out that the difficulties in learning if-then relations stem from uncertainties arising from the cue and target switching between hemifields (as was suggested by one of the reviewers), since in Experiment 2 of our previous paper on this topic (Kristjánsson et al., 2001), observers could not learn a relationship where the target switched from the left side of the cue to the right side from one trial to the next, completely predictably. Observers were, on the other hand, able to learn a consistent location mapping over the course of a few trials (in the streak condition), and performance was quite good in the consistent condition, in which the target was always in the same location relative to the cue within a block of trials. Importantly, in all these three conditions, the cue and the target were moving between hemifields unpredictably, from one trial to the next, and still this strong difference was observed.

4. As was pointed out by one of our referees, our experiments could be taken to mean that when there is a consistent relation between stimuli that transient attention can learn, the claim that our cue was $100 \%$ valid breaks down. The cue validity must, in this case, be defined in terms of whether there is a relationship between cue and target that can be learned and, also, whether the cue-target relationship in each case is consistent or inconsistent with the pattern picked up on by the transient attention mechanism.

5. We are grateful to Dr. Roger Remington for this suggestion.

(Manuscript received February 2, 2001; revision accepted for publication January 7,2003 .) 